\begin{tabular}{|c|c|}
\hline Title & $\begin{array}{l}\text { Structure and Energy Deposition Process of an Inductively Coupled Plasma Under Confronting Divergent Magnetic } \\
\text { Fields }\end{array}$ \\
\hline Author(s) & Minami, Y udai; A sami, Y usuke; Sugawara, Hirotake \\
\hline Citation & $\begin{array}{l}\text { IEEE Transactions on Plasma Science, 42(10), 2550-2551 } \\
\text { https://doi.org/10.1109/T PS.2014.2343275 }\end{array}$ \\
\hline Issue Date & $2014-10-21$ \\
\hline Doc URL & http://hdl.handle.net/2115/57756 \\
\hline Rights & $\begin{array}{l}\text { (c) } 2014 \text { IEEE. Personal use of this material is permitted. Permission from IEEE must be obtained for all other uses, in } \\
\text { any current or future media, including reprinting/republishing this material for advertising or promotional purposes, } \\
\text { creating new collective works, for resale or redistribution to servers or lists, or reuse of any copyrighted component of } \\
\text { this work in other works. }\end{array}$ \\
\hline Tyре & article (author version) \\
\hline File Information & Minami-2014-huscap68067.pdf \\
\hline
\end{tabular}

Instructions for use 


\title{
Structure and Energy Deposition Process of an Inductively Coupled Plasma under Confronting Divergent Magnetic Fields ${ }^{1}$
}

\author{
Yudai Minami, Yusuke Asami, and Hirotake Sugawara ${ }^{2}$
}

\begin{abstract}
We simulated electron motion in confronting divergent magnetic fields (CDMFs) using a Monte Carlo method. Fundamental plasma structure under a filtering effect of the separatrix of CDMFs was depicted. Hot spots of energy deposition into electrons were observed not only near the RF antenna but also near the equi-strength surface of $2 B_{\mathrm{ECR}}$, where $B_{\mathrm{ECR}}$ is the RF-resonant magnetic field strength.
\end{abstract}

Confronting divergent magnetic fields are a characteristic field configuration with a cusp structure to drive so-called X-point plasma used as a negative ion source $[1,2]$. The CDMFs have a filtering effect, which separates the plasma into two parts of different profiles. In order to investigate the separation mechanism and to seek for its applications in plasma processing, e.g. for low-damage material processes, we analyzed electron motion in the CDMFs using a Monte Carlo method. An early phase of the fundamental plasma structure formation was observed as the first step of this investigation.

The model chamber was a cylinder and its diameter and height were $40 \mathrm{~cm}$ (see Fig. 1). The origin of the $r$ and $z$ coordinates was defined at the center of the chamber. The electric field was induced by an RF antenna $(13.56 \mathrm{MHz}, 30 \mathrm{~A})$ on the top of the chamber. The CDMFs, $B$, were applied by two 36-turn coils coaxially surrounding the chamber. The coil currents were $I_{\text {top }}=+33.3 \mathrm{~A}$ and $I_{\text {bottom }}=-100 \mathrm{~A}$. The magnetic null point, or the $\mathrm{X}$ point, was formed at $(r, z)=(0,10.4 \mathrm{~cm})$, at which the $z$-axis crossed the separatrix of the CDMFs. The upward shift of the separatrix was for effective electron acceleration in the upper region. The equi-strength surfaces of $|B|=2 B_{\mathrm{ECR}}$ and $B_{\mathrm{ECR}}$ are shown together in Fig. 1. Here, $B_{\mathrm{ECR}}=2 \pi(\mathrm{m} / e) f_{\mathrm{RF}}$ is the RF-resonant magnetic field strength, $e$ and $m$ are the electronic charge and mass, and $B_{\mathrm{ECR}}=0.48 \mathrm{mT}$ at $f_{\mathrm{RF}}=13.56 \mathrm{MHz}$.

The gas was assumed to be Ar at 5 mTorr. The initial electrons were released at random from the chamber ceiling. Their motion described by position $(x, y$, and $z)$ and velocity $\left(v_{x}, v_{y}\right.$, and $\left.v_{z}\right)$, including free flight, collision with gas molecule, and reflection at the chamber wall, was traced for $50 \mathrm{RF}$ periods and mapped to the $r-z$ plane when sampling under an assumption of azimuthal homogeneity. The Monte Carlo code was capable of treating $1.5 \times 10^{5}$ particles at a time, and a scaling technique was employed to calculate the space charge field.

Fig. 2(a) shows the spatial distributions of electrons $n_{\mathrm{e}}$ and their mean energy $\langle\varepsilon\rangle$. Electrons tended to remain in the upper region because they reciprocated between the

\footnotetext{
${ }^{1}$ Published source: IEEE Transactions on Plasma Science, Vol. 42, No. 10, pp. 2550-2551, October 2014. Manuscript received November 14, 2013; revised May 31, 2014; accepted July 21, 2014. Date of publication August 7, 2014; date of current version October 21, 2014. This work was supported by the Japan Society for the Promotion of Science under Grant 25400528. Digital Object Identifier 10.1109/TPS.2014.2343275

${ }^{2}$ The authors are with the Graduate School of Information Science and Technology, Hokkaido University, Sapporo 060-0814, Japan (e-mail: sugawara@ist.hokudai.ac.jp).
} 


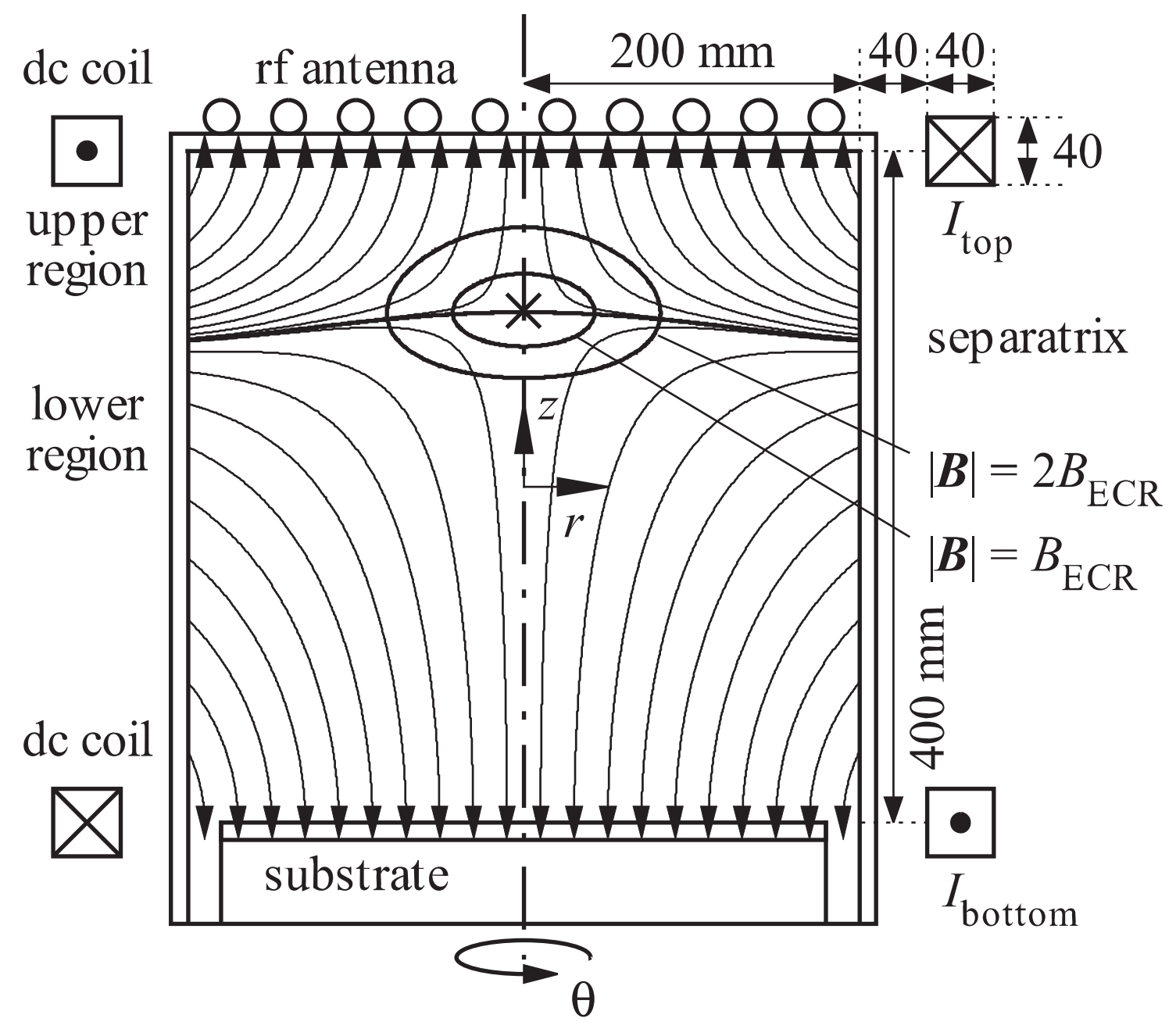

Figure 1: The model reactor and CDMFs. $B=0$ at the $\mathrm{X}$ point. $B_{\mathrm{ECR}}=0.48 \mathrm{mT}$ is the RF-resonant magnetic field strength at $13.56 \mathrm{MHz}$.

chamber ceiling and the side wall being guided by the magnetic field lines and they did not freely diffuse downward across the separatrix. The filtering effect of the CDMFs is understood as this restriction against the downward diffusion. In the lower region, $\langle\varepsilon\rangle$ was relatively high around the $z$-axis and the middle of the three magnetic field lines drawn in Fig. 2(a)(right). A similar profile to the latter was observed also in an experiment [1]. These two areas were reachable from the upper region respectively by electron meandering through the weak magnetic field around the $\mathrm{X}$ point and by diffusion across the separatrix due to scattering near the side wall where the magnetic field lines of the both regions neighbored closely. The latter process is considered to be easier for high-energy electrons than low-energy ones because the displacement of gyro-centers at scattering is large for high-energy electrons correspondingly to their long gyro-radii [3].

Fig. 2(b) shows the electron energy gain $G$ and ionization rate $R_{\mathrm{i}}$. The maximum of $R_{\mathrm{i}}$ coincided not with that of $\langle\varepsilon\rangle$ but with that of the energy density $n_{\mathrm{e}}\langle\varepsilon\rangle$. The filtering effect appeared clearly around the separatrix. $G$ was high near the RF antenna. In addition, such a region was extended to the equi-strength surface of $|B|=2 B_{\mathrm{ECR}}$. The position of $2 B_{\mathrm{ECR}}$ has been regarded as an approximate border between the regions of electron meandering and gyration in gradient magnetic fields $[4,5]$, and electron acceleration by 


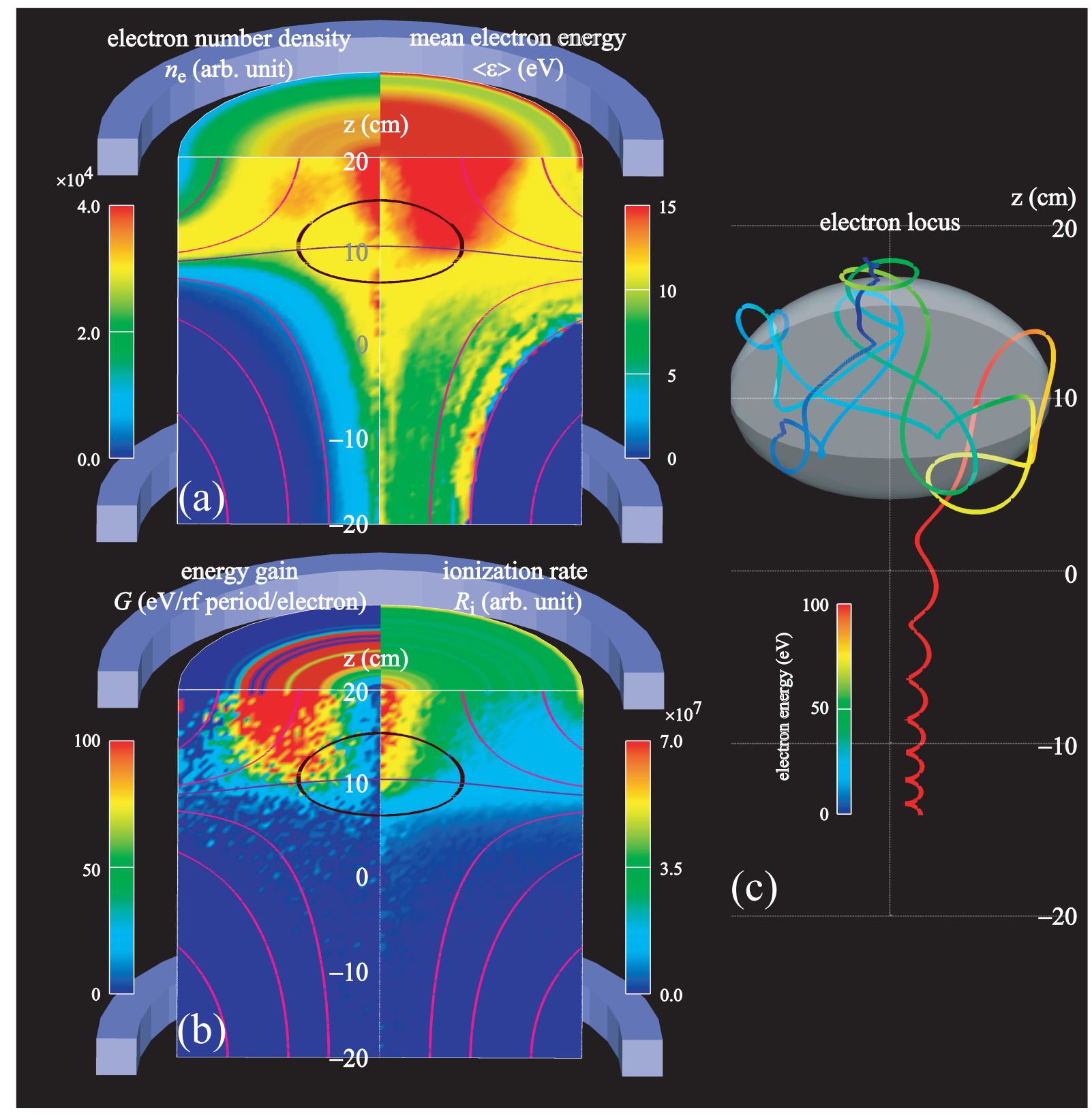

Figure 2: Plasma profiles in CDMFs: (a) time-averaged spatial distributions of electrons $n_{\mathrm{e}}$ and mean electron energy $\langle\varepsilon\rangle$, (b) electron energy gain per RF period per electron $G$ and ionization rate $R_{\mathrm{i}}$, and (c) electron locus and energy around the equi-strength surfaces of $|B|=2 B_{\mathrm{ECR}}$.

the so-called partial Larmor rotation [4] or the partial resonance [6] is expected there. An example electron locus in Fig. 2(c) simulated in a collisionless condition indicates such a steep acceleration around $|B|=2 B_{\mathrm{ECR}}$. Some electrons showed a similar behavior also near $|B|=B_{\mathrm{ECR}}$.

We analyzed electron behavior under the CDMFs. The filtering effect was reproduced and interpreted. The presence of the hot spot of the energy deposition into electrons due to the partial resonance was indicated. 


\section{References}

[1] Ts. Tsankov and U. Czarnetzki, "A discharge with a magnetic X-point as a negative hydrogen ion source," Am. Inst. Phys. Conf. Proc., vol. 1390, pp. 140-149, Nov. 2011.

[2] Ts. Tsankov and U. Czarnetzki, "Hydrogen Discharge with a Magnetic X-Point," IEEE Trans. Plasma Sci., vol. 39, no. 11, pp. 2538-2539, Nov. 2011.

[3] H. Sugawara and Y. Minami, "Monte Carlo simulation of electron motion in confronting divergent magnetic fields," Proc. XXXI Int. Conf. on Phenomena in Ionized Gases, Granada, Spain, 2013.

[4] T. Uchida, "Magnetically neutral loop discharged plasma sources and system," J. Vac. Sci. Technol. A, vol. 16, pp. 1529-1536, May/Jun. 1998.

[5] T. Osaga, H. Sugawara, and Y. Sakurai, "Structure and dynamics of a magnetic neutral loop discharge plasma described using electron motion in a quadrupole magnetic field," Plasma Sources Sci. Technol., vol. 20, no. 6, pp. 065003-1-065003-7, Nov. 2011.

[6] T. Uchida and S. Hamaguchi, "Magnetic neutral loop discharge (NLD) plasmas for surface processing," J. Phys. D, Appl. Phys., vol. 41, no. 8, pp. 083001-1-083001-21, Apr. 2008. 